\title{
ON APPROXIMATION BY EUCLIDEAN AND NON-EUCLIDEAN TRANSLATIONS OF AN ANALYTIC FUNCTION
}

\author{
W. SEIDEL AND J. L. WALSH
}

In 1929 G. D. Birkhoff established ${ }^{1}$ the noteworthy result that an entire function $F(z)$ exists such that to an arbitrary entire function $g(z)$ corresponds a sequence $a_{1}, a_{2}, \cdots$ depending on $g(z)$ with the property

$$
\lim _{n \rightarrow \infty} F\left(z+a_{n}\right)=g(z)
$$

for all $z$, uniformly for $z$ on every closed bounded set.

It is the object of the present note (a) to indicate that not merely an arbitrary entire function $g(z)$ can be expressed in the form (1), but also any function analytic in a simply connected region, and (b) to study the non-euclidean analogue of the entire problem; precisely analogous results are obtained. Some related topics under (a) have recently been studied by $\mathrm{A}$. Roth, ${ }^{2}$ who, however, does not mention the results to be proved here.

The immediate occasion of the interest of the present writers ${ }^{3}$ in the problem is through (b), for non-euclidean translations have been widely used in the study of derivatives of univalent and other functions analytic in the unit circle $|z|=1$; limit functions under such translations are of great significance in the study of derivatives and of limit values of a given function as a variable point $z$ approaches the circumference $|z|=1$.

We shall give a proof of the following theorem, proof and theorem differing only in detail from those of Birkhoff:

THEOREM 1. There exists an entire function $F(z)$ such that given an arbitrary function $f(z)$ analytic in a simply connected region $R$ of the $z$-plane, we have for suitably chosen $a_{1}, a_{2}, \cdots$ the relation

$$
\lim _{n \rightarrow \infty} F\left(z+a_{n}\right)=f(z)
$$

for $z$ in $R$, uniformly on any closed bounded set in $R$.

${ }^{1}$ Comptes Rendus de l'Académie des Sciences, Paris, vol. 189, pp. 473-475.

2 Comentarii Mathematici Helvetici, vol. 11 (1938-1939), pp. 77-125.

${ }^{3}$ Compare Seidel and Walsh, On the derivatives of functions analytic in the unit circle and their radii of univalence and of p-valence, a forthcoming paper in the Transactions of this Society. 
Here and throughout the sequel we are concerned with the finite plane, that is to say, the plane of finite points $z$.

By way of geometric entities, we introduce the circles $C_{1}:|z-4|=2$, $C_{2}:\left|z-4^{2}\right|=2^{2}, \cdots, C_{n}:\left|z-4^{n}\right|=2^{n}, \cdots$, and also the circles $\Gamma_{n}:|z|=4^{n}+2^{n}+1$; it follows that the $C_{n}$ are mutually exterior, and that $\Gamma_{n}$ contains in its interior all the circles $C_{1}, C_{2}, \cdots, C_{n}$ but no point in or on any of the circles $C_{n+1}, C_{n+2}, \cdots$.

Let us enumerate the polynomials in $z$ with rational coefficients: $p_{1}(z), p_{2}(z), \cdots$. It is of course true that any sequence of polynomials can be replaced by a sequence of polynomials with rational coefficients, without altering whatever properties may exist of convergence or uniform convergence to a given function on bounded point sets.

We choose $\pi_{1}(z)$ as a polynomial in $z$ which satisfies the inequality $\left|p_{1}(z-4)-\pi_{1}(z)\right|<1 / 2, z$ on or within $C_{1}$; indeed we may choose $\pi_{1}(z) \equiv p_{1}(z-4)$. We choose $\pi_{2}(z)$ as a polynomial in $z$ which satisfies the two inequalities

$$
\begin{aligned}
\left|\pi_{1}(z)-\pi_{2}(z)\right|<1 / 4, & z \text { on or within } \Gamma_{1}, \\
\left|p_{2}\left(z-4^{2}\right)-\pi_{2}(z)\right|<1 / 4, & z \text { on or within } C_{2} ;
\end{aligned}
$$

such a polynomial $\pi_{2}(z)$ exists, by Runge's classical theorem. In general, let $\pi_{n}(z)$ be a polynomial in $z$ which satisfies the inequalities

$$
\begin{array}{r}
\left|\pi_{n-1}(z)-\pi_{n}(z)\right|<1 / 2^{n}, \quad z \text { on or within } \Gamma_{n-1}, \\
\left|p_{n}\left(z-4^{n}\right)-\pi_{n}(z)\right|<1 / 2^{n}, \quad z \text { on or within } C_{n} .
\end{array}
$$

The sequence $\left\{\pi_{n}(z)\right\}$ converges uniformly in each of the circles $\Gamma_{m}$; hence converges at every point of the plane, uniformly on any bounded set. The limit function $F(z)$ is entire, and has the required properties. Indeed, let $f(z)$ be analytic in a simply connected region $R$; there exist polynomials $p_{n_{k}}(z)$ of the set already defined with

$$
\lim _{n_{k} \rightarrow \infty} p_{n_{k}}(z)=f(z)
$$

at every point of $R$, uniformly on any closed bounded set in $R$. For $z$ in $C_{n}:\left|z-4^{n}\right|<2^{n}$ we have

$$
\begin{aligned}
F(z)= & \pi_{n}(z)+\left[\pi_{n+1}(z)-\pi_{n}(z)\right] \\
& +\left[\pi_{n+2}(z)-\pi_{n+1}(z)\right]+\cdots \\
\left|F(z)-p_{n}\left(z-4^{n}\right)\right| \leqq & \left|p_{n}\left(z-4^{n}\right)-\pi_{n}(z)\right|+\left|\pi_{n+1}(z)-\pi_{n}(z)\right| \\
& +\left|\pi_{n+2}(z)-\pi_{n+1}(z)\right|+\cdots \\
< & \frac{1}{2^{n}}+\frac{1}{2^{n+1}}+\cdots=\frac{1}{2^{n-1}}
\end{aligned}
$$


whence

$$
\lim _{n \rightarrow \infty}\left[F\left(z+4^{n}\right)-p_{n}(z)\right]=0
$$

for every $z$, uniformly on any bounded set. To return to $f(z)$, we now have from (3) and (4)

$$
\lim _{n_{k} \rightarrow \infty}\left[F\left(z+4^{n_{k}}\right)-f(z)\right]=0
$$

for $z$ in $R$, uniformly on any closed bounded set in $R$. Theorem 1 is established.

The special case of Theorem 1 that $f(z)$ is an entire function and $R$ is the (finite) $z$-plane is included here, and is the case considered by Birkhoff. We add the remark that whenever a function $g(z)$ can be represented on a point set $E$ (bounded or unbounded) by a sequence of polynomials, that function can also be represented on $E$ in the manner indicated by (2), with preservation of the property of uniform convergence whenever that occurs on a bounded set belonging to $E$. For instance, $E$ may consist of a sequence of disjoint simply connected regions $R_{1}, R_{2}, \cdots$, with $g(z)$ analytic on $E$; then $g(z)$ can be represented on $E$ either by a sequence of polynomials or, as in (2), with uniform convergence on any closed bounded subset of $E$. On the general subject of representation by polynomials there exist modern researches due to Montel, Walsh, Hartogs and Rosenthal, and Lavrentieff. ${ }^{4}$

A further remark in connection with Theorem 1 is that if the numbers $A_{0}, A_{1}, A_{2}, \cdots$ are arbitrary, there exists a sequence $a_{1}, a_{2}, \cdots$ with the property

$$
\lim _{n \rightarrow \infty} F^{(k)}\left(a_{n}\right)=A_{k}, \quad k=0,1,2, \cdots .
$$

To establish (6) it is sufficient to remark that when $m$ is given, the number $a_{m}$ exists with the property

$$
\begin{aligned}
\left|F\left(z+a_{m}\right)-\left[A_{0}+A_{1} z+\frac{A_{2}}{2 !} z^{2}+\cdots+\frac{A_{m}}{m !} z^{m}\right]\right| & <\frac{1}{2^{m} \cdot m !}, \\
& \text { for }|z| \leqq 1 ;
\end{aligned}
$$

from Cauchy's inequality it then follows that we have $\left|F^{(k)}\left(a_{m}\right)-A_{k}\right|$ $<1 / 2^{m}, k=0,1,2, \cdots, m$; the relation (6) follows.

${ }^{4}$ The reader may refer to Lavrentieff, Sur les Fonctions d'une Variable Complexe Représentables par des Séries de Polynomes, Actualités Scientifiques et Industrielles, no. 441, Paris, 1936. 
We turn now to the non-euclidean analogue of Theorem 1:

THEOREM 2. There exists a function $\Phi(z)$ analytic in the region $|z|<1$ such that given an arbitrary function $\phi(z)$ analytic in a simply connected subregion $R$, we have for suitably chosen $\alpha_{1}, \alpha_{2}, \cdots$ the relation

$$
\lim _{n \rightarrow \infty} \Phi\left(\frac{z+\alpha_{n}}{1+\bar{\alpha}_{n} z}\right)=\phi(z),
$$

for $z$ in $R$, uniformly on any closed set interior to $R$.

As in Theorem 1 we needed to use only real $a_{n}$, so here we shall actually employ only real $\alpha_{n}$.

For geometric entities we choose here $C_{1}$ as the n.e. circle of n.e. radius 2 whose n.e. center is the point $z=\beta_{1}$ of the axis of reals whose n.e. distance from $z=0$ is 4 , and in general choose $C_{n}$ as the n.e. circle of n.e. radius $2^{n}$ whose n.e. center is the point $z=\beta_{n}$ of the axis of reals whose n.e. distance from $z=0$ is $4^{n}$. Let $\Gamma_{n}$ be the circle whose center is $z=0$ and n.e. radius $4^{n}+2^{n}+1$, so that $\Gamma_{n}$ contains in its interior all the circles $C_{1}, C_{2}, \cdots, C_{n}$, but no point in or on any of the circles $C_{n+1}, C_{n+2}, \cdots$.

As before, we use the polynomials $p_{1}(z), p_{2}(z), \cdots$ with rational coefficients. Choose $\pi_{1}(z)$ as a polynomial in $z$ which satisfies the inequality

$$
\left|p_{1}\left(\frac{z-\beta_{1}}{1-\beta_{1} z}\right)-\pi_{1}(z)\right|<1 / 2, \quad z \text { on or within } C_{1}
$$

choose $\pi_{2}(z)$ as a polynomial in $z$ which satisfies the two inequalities

$$
\begin{array}{r}
\left|\pi_{1}(z)-\pi_{2}(z)\right|<1 / 4, \quad z \text { on or within } \Gamma_{1}, \\
\left|p_{2}\left(\frac{z-\beta_{2}}{1-\beta_{2} z}\right)-\pi_{2}(z)\right|<1 / 4, \quad z \text { on or within } C_{2} .
\end{array}
$$

In general, let $\pi_{n}(z)$ be a polynomial in $z$ which satisfies

$$
\begin{array}{r}
\left|\pi_{n-1}(z)-\pi_{n}(z)\right|<1 / 2^{n}, \quad z \text { on or within } \Gamma_{n-1} ; \\
\left|p_{n}\left(\frac{z-\beta_{n}}{1-\beta_{n} z}\right)-\pi_{n}(z)\right|<1 / 2^{n}, \quad z \text { on or within } C_{n} .
\end{array}
$$

The sequence $\left\{\pi_{n}(z)\right\}$ converges uniformly in each of the circles $\Gamma_{m}$, hence converges at every point of the region $|z|<1$, uniformly on any closed subset. The limit function $\Phi(z)$ is analytic throughout the region $|z|<1$, and will now be shown to have the required properties. 
For $z$ in $C_{n}$ we have

$$
\begin{aligned}
\Phi(z)= & \pi_{n}(z)+\left[\pi_{n+1}(z)-\pi_{n}(z)\right] \\
& +\left[\pi_{n+2}(z)-\pi_{n+1}(z)\right]+\cdots, \\
\left|\Phi(z)-p_{n}\left(\frac{z-\beta_{n}}{1-\beta_{n} z}\right)\right| \leqq & \left|p_{n}\left(\frac{z-\beta_{n}}{1-\beta_{n} z}\right)-\pi_{n}(z)\right| \\
& +\left|\pi_{n+1}(z)-\pi_{n}(z)\right|+\cdots \\
< & \frac{1}{2^{n}}+\frac{1}{2^{n+1}}+\cdots=\frac{1}{2^{n-1}},
\end{aligned}
$$

whence

$$
\lim _{n \rightarrow \infty}\left[\Phi\left(\frac{z+\beta_{n}}{1+\beta_{n} z}\right)-p_{n}(z)\right]=0
$$

for every $z$ in $|z|<1$, uniformly on any closed set in $|z|<1$.

Let $\phi(z)$ be analytic in the simply connected region $R$ in $|z|<1$. There exist polynomials $p_{n_{k}}(z)$ of the set already defined with

$$
\lim _{n_{k} \rightarrow \infty} p_{n_{k}}(z)=\phi(z)
$$

at every point of $R$, uniformly on any closed subset. From (8) and (9) we find

$$
\lim _{n_{k} \rightarrow \infty}\left[\Phi\left(\frac{z+\beta_{n_{k}}}{1+\beta_{n_{k}} z}\right)-\phi(z)\right]=0
$$

for $z$ in $R$, uniformly on any closed set in $R$.

Theorem 2 is established. The region $R$ may in particular be the region $|z|<1$. Remarks for Theorem 2 entirely analogous to those for Theorem 1 are also valid.

UNIVERSITY OF ROCHESTER AND

HARVARD UNIVERSITY 